\title{
Ejecución del Programa de Prevención y Control de la Conducta Suicida en Nueva Paz
}

\section{I mplementation of the Prevention and Control Program of Suicidal Behaviours in Nueva Paz municipality}

\author{
MsC. I dalberto Aguilar Hernández,' DraC. I sabel Louro Bernal,"' \\ Lic. Leidis Sandra Perera Milian'"' \\ 'Facultad de Ciencias Médicas. Mayabeque, Cuba. \\ "Escuela Nacional de Salud Pública. La Habana, Cuba. \\ III Policlínico Universitario "Felipe Poey Aloy". Nueva Paz. Mayabeque, Cuba.
}

\section{RESUMEN}

Introducción: el suicidio se ubica entre las 10 primeras causas de muerte en el mundo. La OMS estima que al menos 1110 personas se suicidan cada día y lo intentan cientos de miles, independientemente de la geografía, cultura, religión, etnia, y alerta además, sobre el incremento de las tasas de suicidio como una tendencia creciente sobre todo en la población joven.

Objetivo: valorar la ejecución del Programa de Prevención y Control de la Conducta Suicida en el municipio de Nueva Paz en el año 2010.

Métodos: estudio descriptivo y transversal. Se aplicaron cuestionarios, entrevistas, guías de observación y exploración de conocimientos sobre el Programa por un grupo de 65 trabajadores de la salud. Se establecieron criterios, indicadores y estándares para la evaluación de las variables de estructura, proceso y resultado. Resultados: el suicidio ocupó la séptima causa de muerte en el municipio y los intentos suicidas incidieron en el sexo femenino y en los grupos de 60 años y más y de 10 a 19 años. No existió un adecuado funcionamiento del Centro Comunitario de Salud Mental ni de los Equipos de Salud Mental. No se realizaron actividades de capacitación sobre el Programa y eran insuficientes los conocimientos para ejecutarlo. Las tarjetas de Enfermedades de Declaración Obligatoria carecían de calidad y el flujo de información era inadecuado. Se realizaba una deficiente dispensarización de los pacientes, en lo que incidía la baja calidad de la primera consulta, la no realización de interconsultas y la no periodicidad establecida en las visitas de terreno. 
Conclusiones: las deficiencias encontradas en la estructura y el proceso conllevaron a que la ejecución del Programa de Prevención y Control de la Conducta Suicida en Nueva Paz en el 2010, fuera inadecuada.

Palabras clave: Programa Nacional de Prevención y Control de la Conducta Suicida, atención primaria de salud.

\section{ABSTRACT}

Introduction: suicide is one of the 10 first causes of death worldwide. The World Health Organization estimates that at least one thousand and one hundred ten (1 110) people commit suicide every day and other hundreds of thousands attempted suicide, regardless of geography, culture, religion and race, and this organization also alerts to the increase of suicidal rates as a growing trend in the young population.

Objective: to assess the implementation of the program of prevention and control of suicidal behavior in Nueva Paz municipality in 2010.

Methods: cross-sectional and descriptive study. A group of 65 health workers administered questionnaires, interviews, observational guides and knowledge exploration guides about the Program. Criteria, indicators and standards for the evaluation of structure, process and outcome variables were set.

Results: suicide was the 7th cause of death in the municipality and the attempted suicides had an incidence on the females and on the 60 years and over group and on the 10-19 years-old group. Neither the community center of mental health nor the mental health teams worked properly. Training activities on the program were not carried out and the knowledge for its implementation was really poor. The compulsory notification disease cards lacked good quality and the flow of information was inadequate. The classification of patients was deficient due to low quality of the first doctor's consultation, the non compliance with inter-consultation schedules and the irregular visits paid to the patients' houses Conclusions: the problems found in the structure and in the process led to the negative evaluation of the implementation of the program of suicidal behavior prevention and control in Nueva Paz municipality.

Key words: National Program of Prevention and Control of suicidal behavior, primary health care.

\section{NTRODUCCI ÓN}

La OMS plantea que cada 42 seg alguien hace un Intento suicida con ocurrencia del suceso cada 17 min sobre todo en jóvenes entre 5 y 14 años de edad, por lo que alerta sobre el incremento de las tasas de suicidio como una tendencia creciente, sobre todo, en esta población. Los jóvenes, en determinado momento de su existencia, sienten que la vida no tiene sentido para ellos, ya sea por la presencia de enfermedades físicas o mentales, la pérdida de una relación valiosa, un embarazo oculto o no deseado o las dificultades cotidianas de un mundo convulso, por lo que el suicidio se convierte en su mejor y única opción. ${ }^{1,2}$ El suicidio se ubica 
entre las 10 primeras causas de muerte en el mundo según la OMS, la cual estima que al menos 1110 personas se suicidan cada día y lo intentan cientos de miles, independientemente de la geografía, cultura, religión o etnia.

En los países desarrollados de Europa y América del Norte, el suicidio figura entre la quinta y décima causa de defunción más importante y aparece como segunda o tercera causa entre personas de 15 a 44 años de edad. En trabajos realizados en diferentes partes del mundo se encuentra que la frecuencia del suicidio es mayor en los hombres que en las mujeres mientras que el intento suicida es mayor en mujeres que en hombres. Según informes, se producen entre 10 y 15 intentos suicidas por cada suicidio que ocurre, siendo mayor la relación en los niños y adolescentes. ${ }^{3,4}$

Este fenómeno que va en aumento en todos los países del mundo, se encuentra considerado ya como parte de "Las enfermedades del desarrollo", así tenemos por ejemplo más de 8000 casos en el año 1992 en los Estados Unidos comprendidos entre las edades de 15 a 24 años, ${ }^{5}$ al igual que en Francia, donde el incremento ha alcanzado cifras alarmantes. ${ }^{6}$

En Cuba, el suicidio se ha convertido en un daño importante a la salud. Era el país de más alta tasa en América Latina en el año 2005. Las lesiones autoinfligidas intencionalmente en el 2006 y 2007 ocuparon la décima causa de las muertes de todas las edades con 1376 y 1296 defunciones, respectivamente, las cuales representan una tasa de 12,2 y 11,5 por 100000 habitantes. $^{7}$

La provincia de La Habana se ha mantenido en igual comportamiento e incluso en ocasiones ha tenido tasas superiores a la media nacional como sucedió en el 1995 que alcanzó 23,3 x 100000 habitantes y en el 2004 se reportó una tasa de 15,8 x 10000 habitantes, encontrándose entre la sexta y séptima causa de muerte.

En el municipio habanero de Nueva Paz la conducta suicida constituye uno de los principales problemas de salud desde el año 1990, aportando en el año 1996 una tasa de 37,7 x 100000 habitantes y en estos últimos tres años se muestran tasas superiores a $34,0 \times 100000$ habitantes, cifra que no corresponde con la tasa esperada de países en vías de desarrollo y además, el total de intentos y suicidios no cumplen la razón esperada.

En este territorio se realizó una investigación que abarcó un estudio desde el 1994 hasta el 2004, en el cual se demuestra el comportamiento epidemiológico de dicho daño a la salud. Se concluye que predomina el grupo de 20 a 29 años, el sexo femenino, los estudiantes, nivel escolar medio, el estado civil soltero y residencia urbana. Prevalece como antecedente patológico personal los trastornos psiquiátricos depresivos y en más de la mitad, la presencia de intento suicida en los familiares. Más del $50 \%$ de los pacientes emplea como método la ingestión de psicofármacos.

Se encuentra que el intento suicida predomina en familias disfuncionales y severamente disfuncionales, con el 64,53 y el $7,03 \%$, respectivamente. ${ }^{8}$

En el 2006 se realizaron tres actividades de capacitación dirigidas al personal de salud en la adecuada conducción del Programa Nacional de Prevención y Control de la Conducta Suicida, con énfasis en las acciones de prevención y promoción necesarias para su prevención; a pesar del descenso de las estadísticas municipales relacionadas con los casos de intento suicida, continua siendo uno de los principales problemas de salud en Nueva Paz. Se explora el nivel de conocimientos que posee el personal médico que labora en los servicios de urgencias para el manejo de esta 
urgencia psiquiátrica y se encuentra un déficit de conocimientos para el abordaje de esta entidad de manera integral. ${ }^{9}$

Teniendo en cuenta lo anteriormente planteado, se decidió realizar esta investigación con el objetivo de evaluar la ejecución del Programa Nacional de Prevención y Control de la Conducta Suicida en Nueva Paz.

\section{MÉTODOS}

Se realizó un estudio descriptivo y transversal sobre el Programa de Prevención y Control de la Conducta Suicida en el municipio de Nueva Paz en el año 2010.

El grupo de investigación estuvo constituido por 65 trabajadores de la salud, 23 médicos y 30 enfermeras de consultorios médicos de la familia (CMF), 3 técnicos de recursos humanos, 3 técnicos de estadísticas y 6 integrantes del Centro Comunitario de Salud Mental (CCSM) que se encontraban laborando en el municipio cuando se realizó el estudio.

Se aplicaron cuestionarios a trabajadores del departamento de recursos humanos, CCSM, estadísticas, miembros de los equipos básicos de salud. Además se emplearon guías de revisión documental para las historias clínicas individuales, familiares, tarjetas de Enfermedad de Declaración Obligatoria (EDO) y actas de reuniones de la Comisión de Salud Mental.

Se aplicó un cuestionario de identificación de necesidades de aprendizaje a médicos y enfermeras de la familia y trabajadores del CCSM sobre el Programa Nacional de Prevención y Atención a la Conducta Suicida, validado por expertos. Todo ello responde a una evaluación de estructura, proceso y resultados.

\section{Variables de estructura}

Trabajadores en función del programa según categoría ocupacional, preparación del personal para enfrentar el programa, existencia, composición y funcionamiento de la Comisión de Salud Mental y el llenado correcto o incorrecto de registros y tarjetas de EDO.

\section{Variables de proceso}

Calidad de las actas de reuniones de la Comisión de Salud Mental, de las actividades educativas y discusiones de caso así como el cumplimiento del flujo de información, dispensarización y seguimiento de los casos con intento suicida.

\section{Variables de resultado}

Incidencia de intento suicida y fallecidos por Conducta suicida en los diferentes grupos de edades.

A cada criterio se le fijó un indicador y un estándar. Para declarar la ejecución del programa como adecuada debe existir como mínimo el $70 \%$ de los criterios

http://scielo.sld.cu 
establecidos con su estándar cumplido. Estos se fijaron según la opinión de expertos.

El procesamiento de los datos se realizó por medios computarizados y los datos se expresaron en valores absolutos y relativos.

Para la realización de la investigación se tuvo en cuenta el consentimiento informado y la disposición de los involucrados. Se tuvo en cuenta el cumplimiento de los aspectos éticos en todos los momentos de la investigación.

\section{RESULTADOS}

En el municipio de Nueva Paz el suicidio ocupó el séptimo lugar como causa de mortalidad en el 2010. Durante este año hubo un total de 8 defunciones del sexo masculino y el ahorcamiento fue la causa en el $100 \%$ de los fallecidos. Tuvo una mayor incidencia el área de salud de Los Palos con el $75 \%$ de suicidios (6 personas). El $50 \%$ de los casos fueron mayores de 60 años de edad. El intento suicida aportó 66 pacientes con 69 intentos registrados en ese año; 3 de estos pacientes realizaron 2 intentos en el año. Las féminas, con 45 casos para el 68,14 $\%$,contribuyeron con el mayor número de intentos suicidas. Estas lesiones autoinfligidas tuvieron una mayor incidencia en el grupo de mayores de 60 y de 10 a 19 años con 15 y 14 casos para el 22,71 y $21,21 \%$, respectivamente.

En el año 1998 se acreditó en este municipio el CCSM con una plantilla completa, la que fue decreciendo hasta solo contar con una licenciada en enfermería, una defectóloga, una psicometrista, una trabajadora social y una terapeuta ocupacional que funcionaban como Equipo de Atención a la Salud Mental liderados por la Vicedirectora de Asistencia Médica Municipal. Durante esta etapa se ofrecían consultas de psiquiatría por un médico general en función de psiquiatra que daba cobertura asistencial pero con la insatisfacción de que la sobrecarga asistencial no le permitía cumplir los programas de salud.

A partir de abril del 2010, se incorpora en el territorio una psiquiatra general, en el mes de septiembre una psicóloga y se conforma el Grupo Operativo de Atención a la Salud Mental y las Adicciones (GOSMA) en el municipio, con la psiquiatra como responsable de este equipo y lidera el CCSM. Se cuenta además para la atención a este programa con dos psicólogas, tres trabajadores sociales y 24 equipos básicos de salud en consultorios necesarios, con un total de 22 médicos y 24 enfermeras. Componen además la Comisión Municipal de Salud Mental una enfermera responsable de la educación para la salud, un epidemiólogo y un técnico en estadística. En las áreas de salud no funcionaban los Equipos de Salud Mental (ESM) por falta de recursos humanos para el completamiento de esta estructura.

En la etapa que evaluamos no se desarrolló ninguna actividad de capacitación relacionada con el Programa Nacional de Prevención y Control de la Conducta Suicida. En el cuestionario y entrevistas aplicada a los 21 médicos, 25 enfermeras de la familia y 6 trabajadores del CCSM, para explorar los conocimientos sobre el Programa Nacional se obtuvieron los siguientes resultados:

- El 53,85 \% de los encuestados no poseía ningún conocimiento, el 26,92 \% tenía conocimientos insuficientes y solo el 19,23 \% tenían algunos conocimientos para conducir el Programa. 
- Las principales debilidades en el área del conocimiento estaban dadas por la inadecuada evaluación del intento suicida, insuficiente identificación de los factores de riesgo individuales, familiares y comunitarios, inadecuada clasificación de los métodos utilizados e insuficiente identificación de las acciones a realizar por el equipo básico de salud

- El 71,74 \% de los médicos y enfermeras de la familia encuestada manifestaron no poseer en su consultorio el Programa Nacional de Prevención y Control de la Conducta Suicida y el 100 \% no había recibido capacitación alguna, por lo que no se consideran aptos para cumplimentar las acciones correspondientes al mismo.

- Los miembros del equipo básico de salud alegaron demora en conocer los pacientes que cometen el intento suicida. Al no existir modelo oficial, no llegaba al CMF la tarjeta de EDO, se archivaba en el Departamento de Estadística Municipal.

En los departamentos de estadísticas de las áreas de salud y a nivel municipal existían los registros de EDO con calidad y tenían los datos de la mortalidad por suicidio y la morbilidad por intento suicida. En el $45 \%$ de los casos existieron dificultades en el llenado correcto de las tarjetas de EDO, entre ellas, ausencia de segundo apellido, número de CMF, dirección particular incompleta y no especificación del método empleado para el intento aún después de tantos meses.

En el año 2010 se planificaron 12 reuniones de la Comisión Municipal de Salud Mental y se efectuaron 7 de ellas $(58,33 \%)$. Esta comisión estuvo liderada durante esta etapa por la Vicedirectora de Asistencia Médica Municipal, quien se mantuvo laborando asistencialmente durante 5 meses en un consultorio médico rural. Las actas de la Comisión Municipal se revisaron en su formato y contenido evaluándose de aceptable aunque es de señalar que hubo poca discusión de los temas abordados y poca profundidad en las acciones propuestas. No se discutían sistemáticamente los fallecidos y nunca se evaluó el Programa con informes de comportamiento por áreas de salud. No hubo presencia de invitados para asumir la intersectorialidad del programa.

En entrevista a profundidad realizada a la Vicedirectora de Asistencia Médica Municipal y a la psiquiatra del CCSM, se obtuvieron las siguientes opiniones:

- El CCSM se creó en el 1998 pero funcionaba hasta ahora como un servicio de salud mental.

- El déficit de recursos humanos ha incidido en el completamiento del CCSM y de los ESM de las áreas de salud para la ejecución del programa.

- No se realizan discusiones de casos en las reuniones de los grupos básicos de trabajo.

- Estamos en un proceso de organización y completamiento de la plantilla del CCSM, quien lidera el programa municipalmente.

- Urge la necesidad de capacitación a trabajadores del sector de la salud, promotores comunitarios, organismos involucrados y el adiestramiento a jóvenes y grupos vulnerables.

- No existe una estrategia de comunicación social ante este problema de salud en el municipio. 
Además, se pudo constatar la no utilización de la Casa de Cultura y la Casa de Orientación a la Mujer y la Familia, como medio para la adquisición de conocimientos, actitudes y valores.

Cuentan en la Dirección Municipal con 31 encuestas a pacientes y familiares que asistieron a la consulta de la psiquiatra y que se encontraban satisfechos.

Las tarjetas de EDO que se confeccionan en el servicio de urgencia llegaban al departamento de estadísticas del área de salud de forma diaria en la entrega de guardia y con $48 \mathrm{~h}$ de entrega como promedio las que se confeccionaban en el CMF y en el CCSM. Se tenía establecido el parte diario de los casos declarados a la Unidad Municipal de Higiene y Epidemiología (UMHE) y de forma semanal se enviaban estas tarjetas al departamento de estadísticas de la Dirección Municipal de Salud donde se archivaban. El tiempo transcurrido desde que se produjo el intento suicida y se confeccionaba la tarjeta de EDO de los pacientes que atendidos en los servicios de urgencia o en la consulta de psiquiatría y se ponía en conocimiento del equipo básico de salud, promediaba 30 días. No había en el consultorio médico tarjeta de EDO.

En la revisión de las historias clínicas familiares se constató que solo el 31,81 \% de los intentos suicidas $(n=21)$ se encontraban dispensarizados adecuadamente. Como principales dificultades se detectaron que la dispensarización individual carecía de evaluación periódica adecuada y no se registraba la dispensarización familiar con la consiguiente intervención.

En el proceso de revisión documental de las historias clínicas individuales se encontró que el $65,15 \%$ de los pacientes con Intento suicida $(n=43)$ no estaban dispensarizados en el grupo adecuado. La primera consulta de evaluación de riesgo, carecía de calidad en el 59,09 \% $(n=39)$ y se consideró como inadecuada la estrategia de seguimiento. El $100 \%$ de los pacientes no tenían interconsultas con psiquiatría y psicología, no aparecía la valoración integral de la Comisión de Salud Mental. Las visitas de terreno realizadas no cumplieron la periodicidad establecida y carecían de las orientaciones dirigidas a los factores de riesgo y conducta a seguir.

Los miembros de los equipos básicos de salud refirieron que muchos de los pacientes declarados como intento suicida en sus diversas formas clínicas de presentación, fueron diagnosticados por la psiquiatra en su consulta y tuvieron seguimiento, pero no constaba por escrito en ningún documento legal de estos pacientes las evaluaciones realizadas, lo que demostró la no realización de interconsultas.

\section{DISCUSIÓN}

En el municipio Playa de la Ciudad de la Habana en el año 1995 se desarrolló una evaluación integral sobre salud y luego del análisis de su situación de salud, se encuentra que uno de los principales problemas es el suicidio, razón por la que se decide realizar una investigación que tiene como objetivo evaluar algunos aspectos del Programa Nacional de Prevención y Control de la Conducta Suicida. Con un diseño similar al que aquí se expone, se encuentra que el sexo masculino tiene mayor riesgo de morir y que los grupos afectados son los de 50-64 y de 65 años y más. El intento suicida se comporta con mayores tasas para el sexo femenino y los grupos de edades con mayor incidencia lo ocupan el grupo de 50 a 64 años para ambos sexos seguido del de 15-24 para el femenino (Armas Rojas NB, Ramos Valle 
I, Murla Alonso PA, Sosa Lorenzo I, Rodríguez Salva A. Evaluación de algunos aspectos del Programa Nacional de Prevención de la Conducta Suicida. Municipio Playa, 1995).

Estudios similares relacionados con los Intentos suicidas realizados en Inglaterra arrojan que el $73 \%$ de estos correspondían al sexo femenino, e incluso en personas con una alta frecuencia de recidivas en períodos cortos; en Francia, estudios realizados ofrecen datos semejantes. ${ }^{10-12}$

Otro estudio efectuado en el año 2010 en la provincia de Santiago de Cuba sobre la calidad en la ejecución del Programa de Prevención y Control de la Conducta Suicida, diseñado sobre las dimensiones de desempeño científico-técnico y la satisfacción de los usuarios, constata que la calidad en la ejecución del Programa en el policlínico "Mario Muñoz Monroy" resulta inadecuada, puesto que de un total de 42 criterios para evaluar la estructura, el proceso y los resultados, solo $5(11,9 \%)$ fueron adecuados, cifra muy inferior al estándar establecido de 85,7\% por el grupo de expertos. ${ }^{13}$

Algunos autores opinan que no siempre una estructura adecuada presupone resultados favorables, pues esta por sí sola no garantiza el éxito. El hecho de disponer de los recursos humanos, con un alto nivel de competencia, en cantidad suficiente y debidamente calificados, en los lugares y momentos oportunos, constituye la parte decisiva de la calidad de los servicios. ${ }^{14}$

Los objetivos que persigue el Programa de Prevención y Control de la Conducta Suicida son la reducción de la morbilidad por Intento suicida y los indicadores de mortalidad por Suicidio consumado, por lo que se hace necesario que el personal de salud esté debidamente entrenado en la identificación de individuos y familias con factores de riesgo para la Conducta suicida; de esta manera se cumplirá con la finalidad de evitar que se cometa el primer intento, el desenlace fatal y los intentos repetidos. En esta investigación se encontró desconocimiento del personal de salud sobre aspectos fundamentales del Programa, hecho que resulta alarmante, pues la principal actividad del médico y la enfermera de la atención primaria es la prevención. Estos hallazgos coincidieron con los de Blanca Casas y Guibert Reyes. ${ }^{15}$

No debemos olvidar que en cuanto a la prevención de la Conducta suicida, las preocupaciones no deben ser solo sobre el aspecto del tratamiento sino fundamentalmente en los factores de riesgo que contribuyen a poner de manifiesto estas conductas, así como la promoción de una franca comunicación entre los hombres y su autoestima, donde los diferentes elementos de la sociedad pueden y deben desempeñar una función esencial. ${ }^{9,16}$

Podemos concluir que las deficiencias encontradas en la estructura y el proceso conllevaron a que la ejecución del Programa de Prevención y Control de la Conducta Suicida en el municipio Nueva Paz en el 2010, se evaluara como inadecuada. Tales deficiencias son los elementos esenciales que trajeron por consecuencia que los objetivos del Programa no se cumplieran en el período analizado, y donde la competencia y el desempeño médico debieron jugar una función fundamental. Se hace necesario perfeccionar el flujo de información. 


\section{REFERENCI AS BI BLI OGRÁFICAS}

1. Lester D. The monthly distribution of suicides and urbanization. Percept Mot Skills. 1998; 86(3-2): 1458.

2. Pérez Barrero S. El suicidio, comportamiento y prevención. Rev Cubana Med Gen Integr. 1999; 15(2): 196-217.

3. Riera Betancourt C, Alonso Betancourt O, Marro O. La conducta suicida y su prevención. La Habana: Editorial Ciencias Médicas; 2000.

4. Álvarez Sintes R. Atención familiar. En: Álvarez Sintes R, editor. Temas de Medicina General Integral. Vol 1. La Habana: Editorial Ciencias Médicas; 2001. p. 209-79.

5. Riera C, Alonso O, Madrid E. La conducta suicida y su prevención. La Habana: Editorial Ciencias Médicas; 1995.

6. Lecomte Fornes P. Suicide among youth and young adults, 15 through 24 years of age. Paris. J Forensic Sci. 1998;43(5):964-8.

7. Ministerio de Salud Pública. Colectivo de autores. Anuario Estadístico de Salud 2007. La Habana: MINSAP; 2008.

8. Aguilar Hernández I, Perera Milian LS, Esquivel Rodríguez D, Barreto García ME. Comportamiento del intento suicida en el municipio de Nueva Paz. Revista de Ciencias Médicas La Habana [Internet]. 2008[citado 2010 Ago 31];14(3). Disponible en: http://www.cpicmha.sld.cu/hab/vol14_3_08/hab02308.html

9. Aguilar Hernández I, Perera Milian LS, Cañizares Carmenate I. Conocimientos del personal médico en el manejo del intento suicida en los servicios de urgencias. Revista de Ciencias Médicas La Habana [Internet]. 2008[citado 2010 Ago 31]; 14(2) Disponible en: http://www.cpicmha.sld.cu/hab/pdf/vol14_2_08/hab02208.pdf

10. Lester D. Judging the sex and age of suicide note writers. Percept Mot Skills. 1998; 86(3-2): 1218.

11. Mzelewz S, Jonsson K, Aberg M. A prospective study on the epidemiology of burns in patients admitted to the Harare burn units. Burns. 1999;25(6):499-504.

12. Pochard F, Robins M, Fassel Al. Suicidal action and sacrificial violence systemic and anthropological hypothesis. Encephale.1999;25(4):296-303.

13. Soler Santana Raxsy, Castillo Núñez B, Brossard Cisnero M, Peña García CE. Calidad en la ejecución del Programa de Prevención y Control de la Conducta Suicida. MEDISAN [Internet]. 2010 Jul [citado 2010 Ago 31]; 14(5): Disponible en: http://bvs.sld.cu/revistas/san/vol_14_5_10/san10510.htm

14. Asociación Regional de Facultades y Escuelas de Enfermería. Memorias VIII Congreso Nacional de Intercambio Académico. Enfermería hacia el siglo XXI. México, D.F.: Calidad Total; 1995. 
15. Blanca Casas SM, Guibert Reyes W. Identificación de indicadores suicidas en pacientes generales por la enfermera de atención primaria. Rev Cubana Enfermer [Internet]. 1998 [citado 25 Ene 2009];14(2):117-23. Disponible en: http://bvs.sld.cu/revistas/enf/vol14_2_98/enf07298.htm

16. Blumental SJ. El Suicidio: guía sobre factores de riesgo, valoración y tratamiento de los pacientes suicidas. Clin Méd N Amer. 1988; 4:999-1035.

Recibido: 2 de marzo de 2011.

Aprobado: 11 de octubre de 2011.

I dalberto Aguilar Hernández. Calle 4 No. 2108 e/ 21 y 23 Nueva Paz. La Habana, Cuba. Correo electrónico: idaguilar@infomed.sld.cu 